\title{
Quick Recognition of Rock Images for Mobile Applications
}

\author{
Chunling Wang ${ }^{1}$, Yan Li ${ }^{1}$, Guangpeng Fan ${ }^{1}$, Feixiang Chen ${ }^{1, *}$ and Wei Wang ${ }^{2}$ \\ ${ }^{1}$ School of Information Science \& Technology, Beijing Forestry University, Beijing 100083, China \\ ${ }^{2}$ College of Engineering, San Diego State University, San Diego, CA 92182, United States
}

Received 7 April 2018; Accepted 24 September 2018

\begin{abstract}
Rock lithology identification is an important aspect of field geological surveys. However, traditional identification methods cannot obtain real-time effective feedback, a limitation that is ineffective for further implementation of field geological surveys. To obtain rock lithology information quickly in field geological surveys, an automatic identification method of rock lithology applicable to field offline conditions was proposed in this study. Based on MobileNets, a lightweight deep neural network with depthwise separable convolutions, and the transfer learning method, the proposed method was employed to establish a lithology recognition model for rock images. Its applications on mobile devices were verified, and the fast and accurate lithology identification of rock images was realized under the field offline conditions. Results demonstrate that the constructed model achieves $95.02 \%$ identification accuracy on the validation dataset and 93.45\% identification accuracy on the test dataset of mobile devices. The average recognition time of each image is 1186 ms, and images which have result confidence higher than $95 \%$ respectively account for $91 \%$ of the test dataset. The mode size is $17.3 \mathrm{MB}$. These findings indicate that the model has high identification accuracy, short identification time, and reliable identification results. The proposed method provides good references for the establishment of a geological survey intelligent space and has promising prospects for application in field geological surveys.
\end{abstract}

Keywords: Rock recognition, Deep learning, Transfer learning, Mobile devices

\section{Introduction}

With the development of computer technology, geological surveys have undergone great reforms from the traditional working mode to digital mode and then to the intelligent geological survey system [1]. Rock lithology identification is an important part of modern geological research and a vital aspect of field geological surveys. Therefore, rock lithology identification is an interesting and meaningful research topic. In traditional rock lithology identification method based on images, fresh rock samples are collected and brought to the laboratory during field geological survey. A small piece of rock is cut from the vertical bedding direction of rock samples and ground into $0.03 \mathrm{~mm}$-thick slices. The slice is called the rock thin section [2]. Then, the rock types and structural parameters are identified by experienced geological researchers. In the entire process of identification, many defects are found, such as strong subjectivity, strong uncertainty, long judgment period, and high judgment cost [3].

In recent years, the application of image processing technology in various fields has been deepening, and the automatic recognition of rock images has been greatly developed. Existing studies on automatic recognition of rock images mainly focus on the microscopic image of rock thin sections by using image analysis, feature extraction and other image processing techniques to analyze rock texture, petrofabric [4], rock grain size, rock lithology, and other

\footnotetext{
*E-mail address: fxchen@126.com

ISSN: $1791-2377$ @ 2018 Eastern Macedonia and Thrace Institute of Technology. All rights reserved. doi:10.25103/jestr.114.14
}

issues. The automatic recognition of rock thin sections has solved the problems of strong subjectivity and high judgment cost but failed to solve the problem of long judgment period. In practice, field geological surveys are often carried out under offline conditions. Therefore, acquiring the rock lithology information quickly and accurately to identify rock lithology automatically under field offline conditions is an urgent problem requiring a solution.

This study established an automatic recognition model of rock image on the basis of deep learning. Subsequently, the model was deployed to mobile devices and used to analyze the rock profile images captured by mobile devices to identify rock lithology quickly under field offline conditions.

\section{State of the art}

In recent years, plenty of studies on the automatic recognition of rock images have been conducted. With the use of multi-scale analysis of color texture images, Lepistö [5] classified natural rock texture by combining color information and texture description. The experimental data were divided into four types, and a classification accuracy of $81.3 \%$ was obtained. The use of color information increases the classification accuracy of color images, but its computation complexity is determined by scale and number of color channels, which influence the classification accuracy. Singh et al. [6] extracted useful information from rock images using image processing technology and effectively classified them based on a radial basis function network. The experimental data were divided into three 
classes and showed a classification accuracy of $88.71 \%$. However, extremely complex image processing procedures are necessary to extract effective information. Naresh Singh et al. [7] studied the texture recognition method of rock thin sections based on image processing and multi-layer perceptual neural network and succeeded in achieving basalt texture identification. The accuracy of the three texture classes was $92.22 \%$, which was higher than those in previous studies. However, the classification categories were fewer. In 2013, Cheng et al. [8] studied the automatic pore recognition of rock thin section images based on the probabilistic neural network method, which extracted features and implemented classification test by clustering and PNN. However, lithology identification was not considered. In 2015, Cheng et al. [9] classified 100 rock thin section samples of four classes by studying image features and using support vector machine. The accuracy was $80 \%$, but the model performance was poor. Zhang Cuifen et al. [10] synthesized image color by using eigenvectors of lithologic units. Although this process greatly increased the identifiability of lithologic units of rocks, the remote sensing image identification was inapplicable to field geological investigations. With the development of deep learning in the field of image-intelligent identification [11-14], research on the automatic recognition of rock images based on deep learning is increasing. In 2017, Cheng et al. [15] realized the automatic rock particle size identification of rock thin section image based on convolution neural network. Although the identification accuracy of this method reached $98.5 \%$, the standard convolutional neural network had many parameters and was inapplicable to mobile devices. Moreover, the network avoided the lithology identification problem. $\mathrm{Li}$ et al. [16] trained microscopic images of sandstone by transfer learning and gained a high-precision microscopic image classification model of sandstone thin sections. However, this model showed poor adaptation and was only applicable to sandstone identification. Zhang et al. [17] applied transfer learning in automatic lithology identification and classification of rock images for the first time and effectively identified graphite, phyllite, and breccias. However, the experimental data used natural rock images, and the surface weathering was neglected. The identification accuracy of this method was also sensitive to many factors.

In the above methods, the dataset involved few rock types, which could neither verify the expansibility of the classifier nor meet the demands of quick feedback in field geological investigation. Thus, this study proposes a quick lithology identification method of rock images for mobile devices. This method can identify rock lithology quickly and accurately in the geological survey process under offline conditions following the rock profile images collected by mobile devices. Considering the limited computing and storage resources of mobile and embedded devices, the learning results of MobileNets (a lightweight deep neural network with depthwise separable convolution) on the large dataset ImageNet are transferred to the rock dataset of this study, including 25 classes based on MobileNets and the transfer learning method [18]. After retraining, the generated model is exported and deployed on the Android platform to identify rock lithology rapidly and accurately under field offline conditions.

The remainder of this study is organized as follows. Section 3 describes the principle of the model, constructs a lithologic identification model of rocks that is applicable to mobile devices, and introduces the dataset and data processing mechanism. Section 4 analyzes the model training process and results. Furthermore, the model performance on the test dataset of the mobile device is exhibited, and the applicability of the proposed method on the mobile device is verified. Section 5 summarizes the conclusions.

\section{Methology}

\subsection{Depthwise separable convolution}

Considering the continuous applications of deep neural networks in computer vision, the overall trend of designing the deep neural network structure is to develop a deeper and more complicated network for extraction and fusion of image features, thereby achieving high accuracy [19]. This process implies the involvement of abundant parameters and a large-volume model. For the deep neural network to function in mobile and embedded devices with limited resources, the network structure must be optimized to reduce its occupation of computation and storage resources.

Depthwise separable convolution is a convolution structure designed for mobile and embedded devices. The structure factorizes a standard convolution into a depthwise convolution and a pointwise convolution $(1 \times 1$ convolution kernel) [20]. Depthwise convolution applies the filter to each input channel, and $1 \times 1$ pointwise convolution is used to combine the outputs of the depthwise convolution. As shown in the following figures, Fig.1 is a standard convolution structure, and Fig. 2 is a depthwise separable convolution structure.

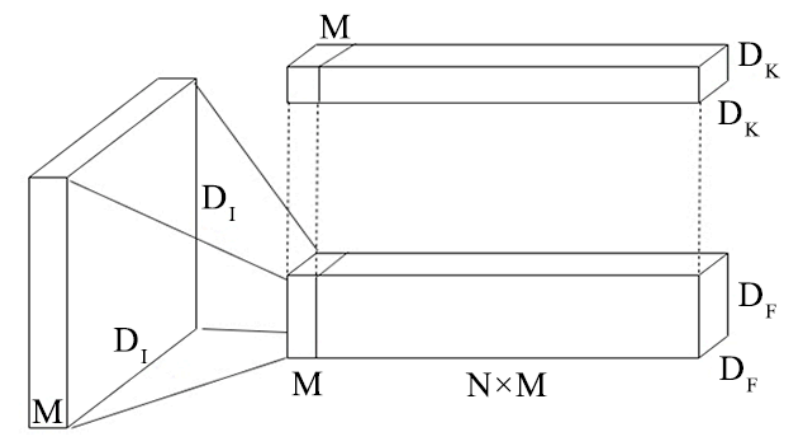

Fig. 1. Standard convolution

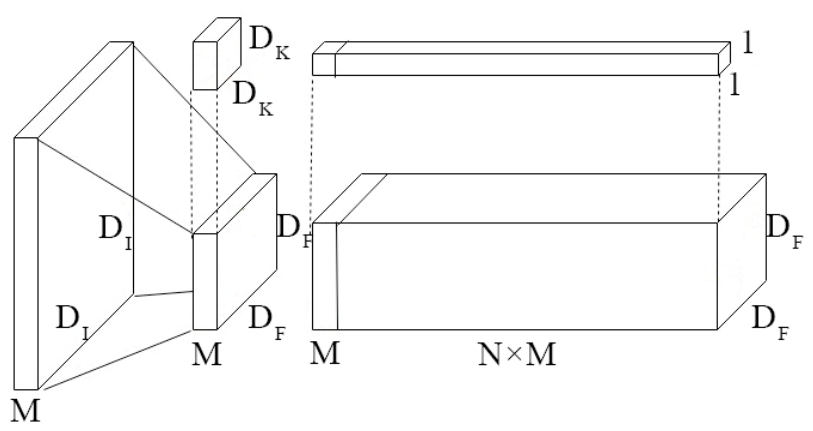

Fig. 2. Depthwise separable convolution

Generally, the connection of neurons is used to represent the computational cost. Standard convolutions (Fig. 1) have the computational cost of $D_{K} \cdot D_{K} \cdot M \cdot N \cdot D_{F} \cdot D_{F}$. Depthwise separable convolutions (Fig. 2) factorize the standard volume integral into two parts: one is depthwise convolution, which has a computational cost of $D_{K} \cdot D_{K} \cdot M \cdot D_{F} \cdot D_{F}$; and the other is pointwise convolution, 
which has a computational cost of $D_{F} \cdot D_{F} \cdot M \cdot N$. After series connection, these two parts consist of a depthwise separable convolution whose computational cost is $D_{K} \cdot D_{K} \cdot M \cdot D_{F} \cdot D_{F}+D_{F} \cdot D_{F} \cdot M \cdot N$

On the basis of the comparison of computation loads between depthwise separable convolution and standard convolution, we obtain

$$
\frac{D_{K} \cdot D_{K} \cdot M \cdot D_{F} \cdot D_{F}+D_{F} \cdot D_{F} \cdot M \cdot N}{D_{K} \cdot D_{K} \cdot M \cdot N \cdot D_{F} \cdot D_{F}}=\frac{1}{N}
$$

This equation shows that the use of $3 \times 3$ depthwise separable convolution kernel $\left(D_{K}=3\right)$ reduces parameters and computation by eight to nine times, whereas the accuracy decreases slightly [20]. Hence, this factorization can effectively decrease computation loads and model size.

\subsection{Transfer learning}

Transfer learning solves learning problems in the target domain by using training data in different but related source domains [21]. The major hypothesis in most machine learning and data mining algorithms is that training data and future (test) data must have the same feature space and distribution. However, in many real applications, this hypothesis may not be valid. In this case, the learning performance can be improved greatly and expensive data marking can be avoided by transferring the knowledge of different domains [22]. The traditional machine learning mode is shown in Fig. 3. Given different learning tasks, different learning systems must be established even if similarities occur between tasks. The transfer learning mode is shown in Fig. 4. To cope with different learning tasks, the knowledge that is learned by the learning system from solving source tasks can be transferred to the learning system that solves the target tasks.

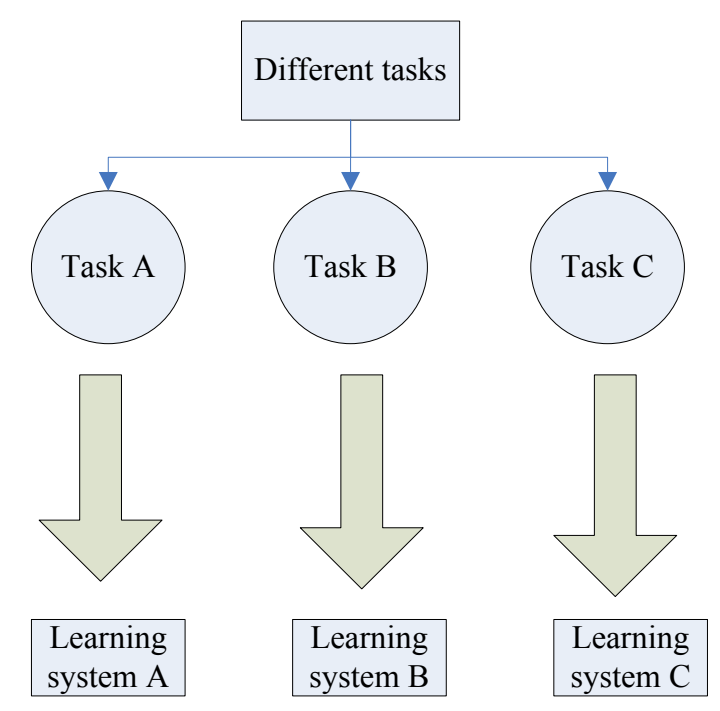

Fig. 3. Traditional machine learning model

From the perspective of the structure and function of deep neural networks, the convolution layer of the network mainly extracts the image features and shares the parameters. The pooling layer is used to reduce the number of parameters, the final full-connection layer integrates the features extracted by the network to obtain the high-level meaning of the image features, and the classifier is used to obtain the final classification result as the final step [23]. In certain cases, the dataset has a small data size and the distribution is uneven because of the limited actual conditions, which may lead to overfitting of training results. Thus, the model performs well in the training set but poorly in the validation set and test set. Transfer learning can address this situation to a certain extent [22].

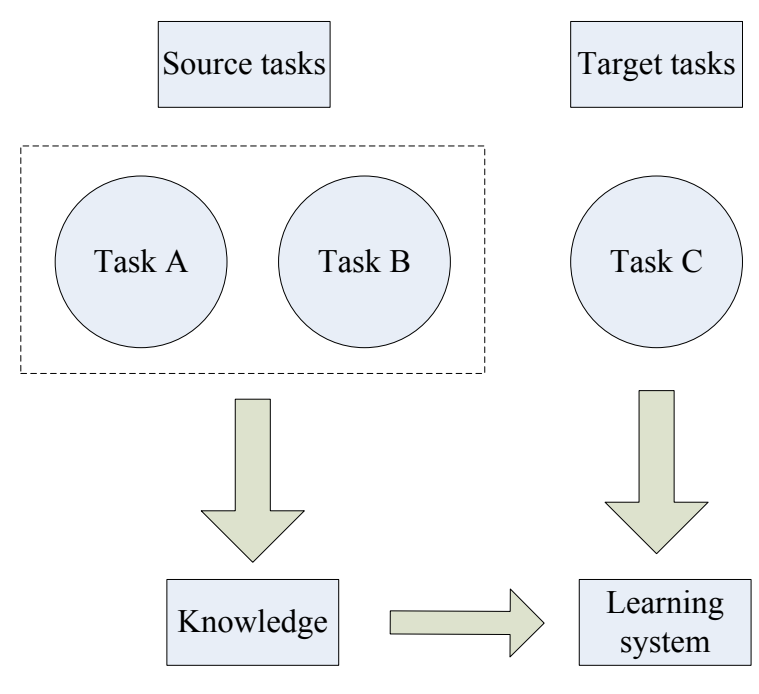

Fig. 4. Transfer learning model

\subsection{Softmax classifier}

Softmax regression algorithm is often used to solve multiclassification problems by mapping the output values of multiple neurons into $(0,1)$, and naturally, their sum is 1 . From this point, the output values can be regarded as the probability that a sample is classified into a certain class.

We consider a training set composed of $m$ marked samples, $\left\{\left(x^{(1)}, y^{(1)}\right), \cdots,\left(x^{(m)}, y^{(m)}\right)\right\}$, where the value range of class label $\mathrm{y}$ is $y^{(i)} \in\{1,2, \cdots, k\}$. We consider that $p(y=j \mid x)$ indicates the probability of samples being classified as class $j$ under the input $\mathrm{x}$. Thus, the output of the $\mathrm{k}$-class classifier is a $\mathrm{k}$-dimensional vector and the sum of elements of this vector is 1 . Similar to logistic regression, the hypothesis function $h_{\theta}\left(x^{(i)}\right)$ can be employed to express the output of Softmax as:

$$
h_{\theta}\left(x^{(i)}\right)=\left[\begin{array}{l}
p\left(y^{(i)}=1 \mid x^{(i)} ; \theta\right) \\
p\left(y^{(i)}=2 \mid x^{(i)} ; \theta\right) \\
\vdots \\
p\left(y^{(i)}=k \mid x^{(i)} ; \theta\right)
\end{array}\right]=\frac{1}{\sum_{j=1}^{k} e^{\theta_{j}^{T} x^{(i)}}}\left[\begin{array}{c}
e^{\theta_{1}^{T} x^{(i)}} \\
e^{\theta_{2}^{T} x^{(i)}} \\
\vdots \\
e^{\theta_{k}^{T} x^{(i)}}
\end{array}\right]
$$

where the input $\mathrm{x}$ is $(\mathrm{m} \times 1)$-dimensional vector and is a parameter of the model, a matrix of $\mathrm{m} \times \mathrm{k}$ order. The training process of the model is to search the optimal $\theta$ by continuous iteration, thereby causing the predicted value to approach the actual value. The cost function of the regression model can be expressed as: 


$$
j(\theta)=-\frac{1}{m}\left[\sum_{i=1}^{m} \sum_{j=1}^{k} 1\left\{y^{(i)}=j\right\} \log \frac{e^{\theta_{j}^{T} x^{(i)}}}{\sum_{l=1}^{k} e^{\theta_{l}^{T} x^{(i)}}}\right]
$$

where $1\{\cdot\}$ is a characteristic function, the rule is $1\{$ value $=$ true $\}=1,1$ value $=$ false $\}=0 . J(\theta)$ is minimized to obtain the value of . As no closed-form solution is available for the minimization of $J(\theta)$, a gradient descent iterative algorithm is available. After derivation, the gradient formula can be gained as follows:

$$
\nabla_{\theta_{j}} J(\theta)=-\frac{1}{m} \sum_{i=1}^{m}\left[x^{(i)}\left(1\left\{y^{(i)}=j\right\}-p\left(y^{(i)}=j \mid x^{(i)} ; \theta\right)\right)\right]
$$

where $\nabla_{\theta_{j}} J(\theta)$ is a vector, and its $l$ th element, $\frac{\partial J(\theta)}{\partial \theta_{j t}}$, is the partial derivative of $\theta_{j}$ to the $l$ component of $J(\theta)$.

In practice, a weight attenuation term is usually added to the cost function $J(\theta)$ to obtain a strict convex function and assure the convergence and a unique solution [24]. Therefore, the new cost function and gradient are

$$
\begin{aligned}
& J(\theta)=-\frac{1}{m}\left[\sum_{i=1}^{m} \sum_{j=1}^{k} 1\{y(i)=j\} \log \frac{e^{\theta_{j}^{T} x^{(i)}}}{\sum_{l=1}^{k} e^{\theta_{l}^{T} x^{(i)}}}\right]+ \\
& +\frac{\lambda}{2} \sum_{i=1}^{k} \sum_{j=0}^{n} \theta_{i j}^{2} \\
& \nabla_{\theta_{j}} J(\theta)=-\frac{1}{m} \sum_{i=1}^{m}\left[x^{(i)}\left(1\left\{y^{(i)}=j\right\}-p\left(y^{(i)}=j \mid x^{(i)} ; \theta\right)\right)\right]+\lambda \theta
\end{aligned}
$$

where $\lambda$ is the weight attenuation coefficient.

\section{Result analysis and discussion}

\subsection{Model architecture}

The inter-class gap in rock image dataset is relatively small compared with a large dataset and is thus considered the fine dataset in specific fields. Rock lithology identification is an example of fine-grained classification. Here, the transfer learning mentioned in Section 3.2 was used for rock image recognition. The network was pre-trained on the large dataset, and most parameters were retained. The retained parameters were adjusted to be applicable to the dataset in this study. With late deployment onto the mobile devices, the lightweight network structure of separable convolution (MobileNets) in Section 3.1 is one solution. The first 28 layers of the convolution structure in MobileNets were frozen. The final full-connection layer was rewritten based on rock image dataset characteristics. MobileNets weights and parameters that were pre-trained on ImageNet dataset were input. Each convolution layer used ReLU as the activation function, and batchnorm was used for the standardized distribution of batches. The Softmax classifier in Section 3.3 was used and rock image dataset was trained again. The convolutional layer structure is shown in Fig. 5, and the model structure is shown in Table 1.



Fig. 5. The structure of convolution unit

Table 1. MobileNets Body Architecture

\begin{tabular}{c|c|c}
\hline Type & Filter Shape & Input Size \\
\hline Conv1 & $3 \times 3 \times 3 \times 32$ & $224 \times 224 \times 3$ \\
Conv2 dw & $3 \times 3 \times 32$ dw & $112 \times 112 \times 32$ \\
Conv2 pw & $1 \times 1 \times 32 \times 64$ & $112 \times 112 \times 32$ \\
Conv3 dw & $3 \times 3 \times 64$ dw & $112 \times 112 \times 64$ \\
Conv3 pw & $1 \times 1 \times 64 \times 128$ & $56 \times 56 \times 64$ \\
Conv4 dw & $3 \times 3 \times 128 \mathrm{dw}$ & $56 \times 56 \times 128$ \\
Conv4 pw & $1 \times 1 \times 128 \times 128$ & $56 \times 56 \times 128$ \\
Conv5 dw & $3 \times 3 \times 128 \mathrm{dw}$ & $56 \times 56 \times 128$ \\
Conv5 pw & $1 \times 1 \times 128 \times 256$ & $28 \times 28 \times 128$ \\
Conv6 dw & $3 \times 3 \times 256 \mathrm{dw}$ & $28 \times 28 \times 256$ \\
Conv6 pw & $1 \times 1 \times 256 \times 256$ & $28 \times 28 \times 256$ \\
Conv7 dw & $3 \times 3 \times 256 \mathrm{dw}$ & $28 \times 28 \times 256$ \\
Conv7 pw & $1 \times 1 \times 256 \times 512$ & $14 \times 14 \times 256$ \\
Conv8 dw & $3 \times 3 \times 512 \mathrm{dw}$ & $14 \times 14 \times 512$ \\
Conv8 pw & $1 \times 1 \times 512 \times 512$ & $14 \times 14 \times 512$ \\
Conv9 dw & $3 \times 3 \times 512 \mathrm{dw}$ & $14 \times 14 \times 512$ \\
Conv9 pw & $1 \times 1 \times 512 \times 512$ & $14 \times 14 \times 512$ \\
Conv10 dw & $3 \times 3 \times 512 \mathrm{dw}$ & $14 \times 14 \times 512$ \\
Conv10 pw & $1 \times 1 \times 512 \times 512$ & $14 \times 14 \times 512$ \\
Conv11 dw & $3 \times 3 \times 512 \mathrm{dw}$ & $14 \times 14 \times 512$ \\
Conv11 pw & $1 \times 1 \times 512 \times 512$ & $14 \times 14 \times 512$ \\
Conv12 dw & $3 \times 3 \times 512 \mathrm{dw}$ & $14 \times 14 \times 512$ \\
Conv12 pw & $1 \times 1 \times 512 \times 512$ & $14 \times 14 \times 512$ \\
Conv13 dw & $3 \times 3 \times 512 \mathrm{dw}$ & $14 \times 14 \times 512$ \\
Conv13 pw & $1 \times 1 \times 512 \times 1024$ & $7 \times 7 \times 512$ \\
Conv14 dw & $3 \times 3 \times 1024 \mathrm{dw}$ & $7 \times 7 \times 1024$ \\
Conv14 pw & $1 \times 1 \times 1024 \times 1024$ & $7 \times 7 \times 1024$ \\
Avg Pool & $\mathrm{P}$ wool 7×7 & $7 \times 7 \times 1024$ \\
FC & $1024 \times 25$ & $1 \times 1 \times 1024$ \\
Softmax & $\mathrm{Classifier}$ & $1 \times 1 \times 25$ \\
\hline
\end{tabular}

4.2 Rock image dataset and preprocess

The rock image dataset in this experiment is a new dataset that consists of fresh rock profile images collected by mobile devices during the field geological survey, including al light grey rhyolite, a2 purple-red tuffite, and 23 other types of different lithologic rock data. All data were collected from multiple locations in East China. The image sizes of the original data vary from $3 \mathrm{M}$ to $5 \mathrm{M}$. To reduce model parameters, the original data were compressed to $224 \times 224$ pixels. The sample images are shown in Fig. 6 as below.

The rock image dataset consists of a total of 3023 images, which belong to 25 categories, of which $80 \%$ were randomly selected as training datasets, $10 \%$ as validation datasets, $10 \%$ as test datasets, 2432 images in the training set, 301 images in the validation set, 290 images the in testing set. The detailed data distribution is shown in Fig. 7. 
Chunling Wang, Yan Li, Guangpeng Fan, Feixiang Chen and Wei Wang/

Journal of Engineering Science and Technology Review 11 (4) (2018) 111 - 117
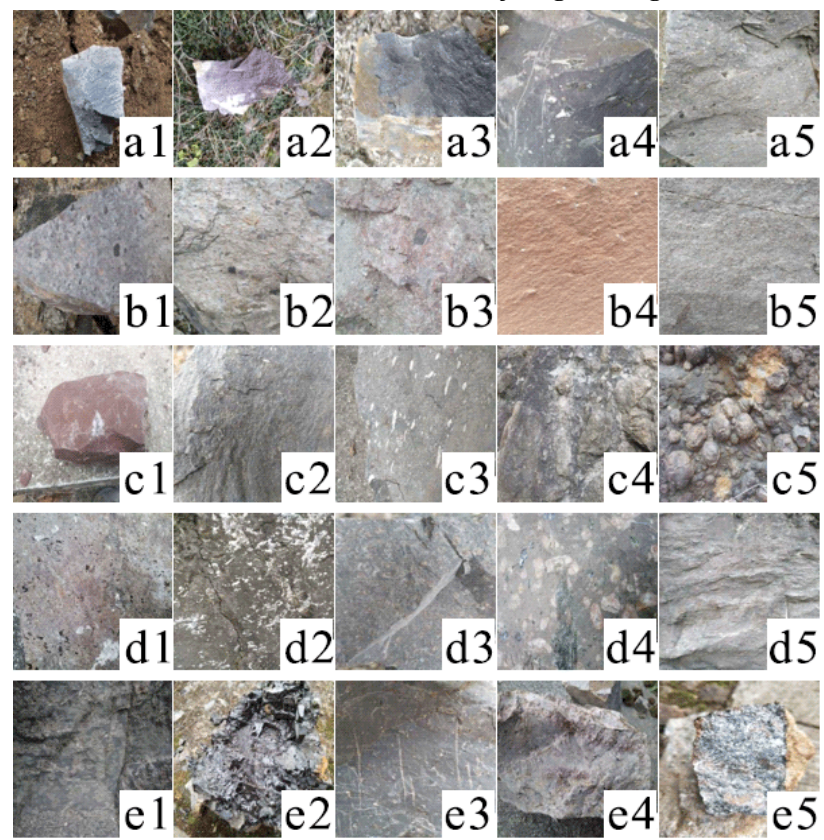

Fig. 6. Samples of data
The number of different classes of the rock image dataset is not balanced, and the number of some classes of pictures is extremely small. The training dataset is augmented by random rotation, flip, cropping, and shading adjustment.

\subsection{Training and analysis}

Model training was started and the MobileNets structure was constructed. Parameters that were trained by MobileNets on the ImageNet dataset were input, and other hyper-parameters were adjusted following experimental performances. The iteration steps were 4,000 , and the learning rate was 0.006 . ReLU was applied as the activation function that was optimized by the stochastic gradient descent method. Loss was verified by cross-entropy, and the classifier used Softmax. In training, 16 images were randomly selected from the dataset in every iteration. The model was evaluated every 20 iterations. Loss and accuracy changes in the first 2,000 iterations in the training process are shown in Fig. 8. Loss began to converge after 200 iterations and began to stabilize after 600 iterations. The loss was nearly 0 and the training accuracy was nearly $100 \%$. The accuracy of the verification dataset fluctuated slightly but approached $100 \%$.

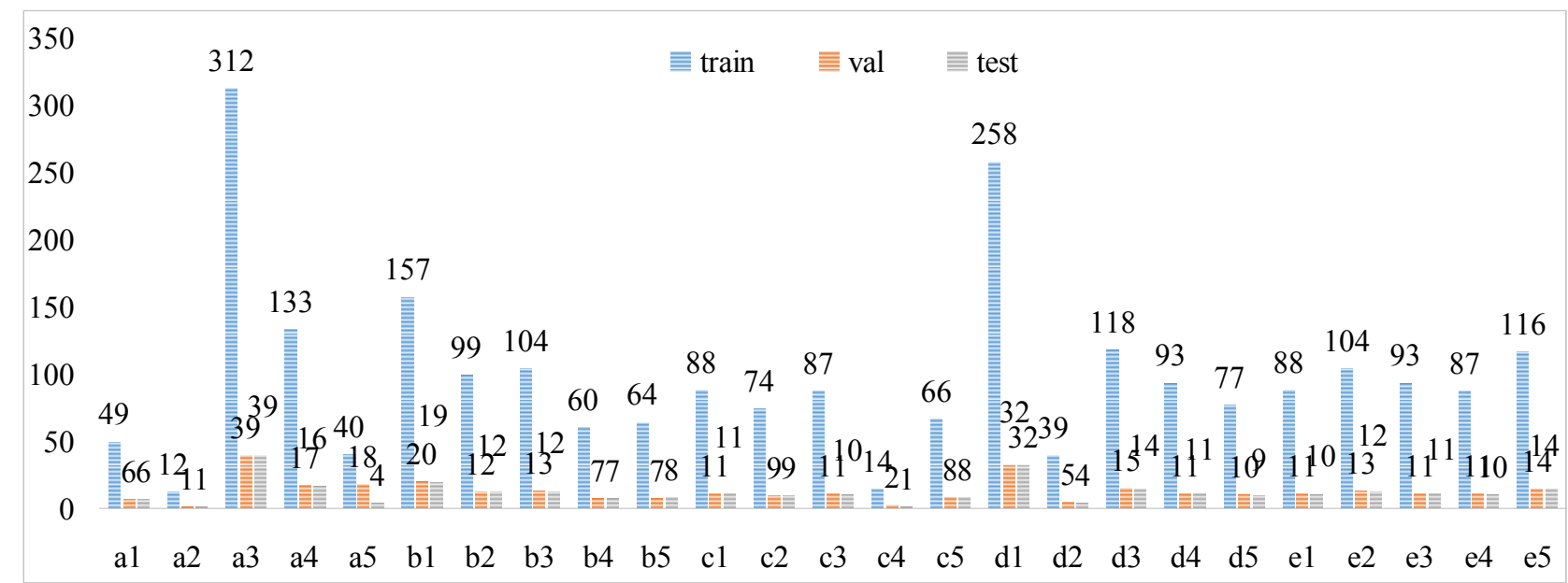

Fig. 7. Data distribution

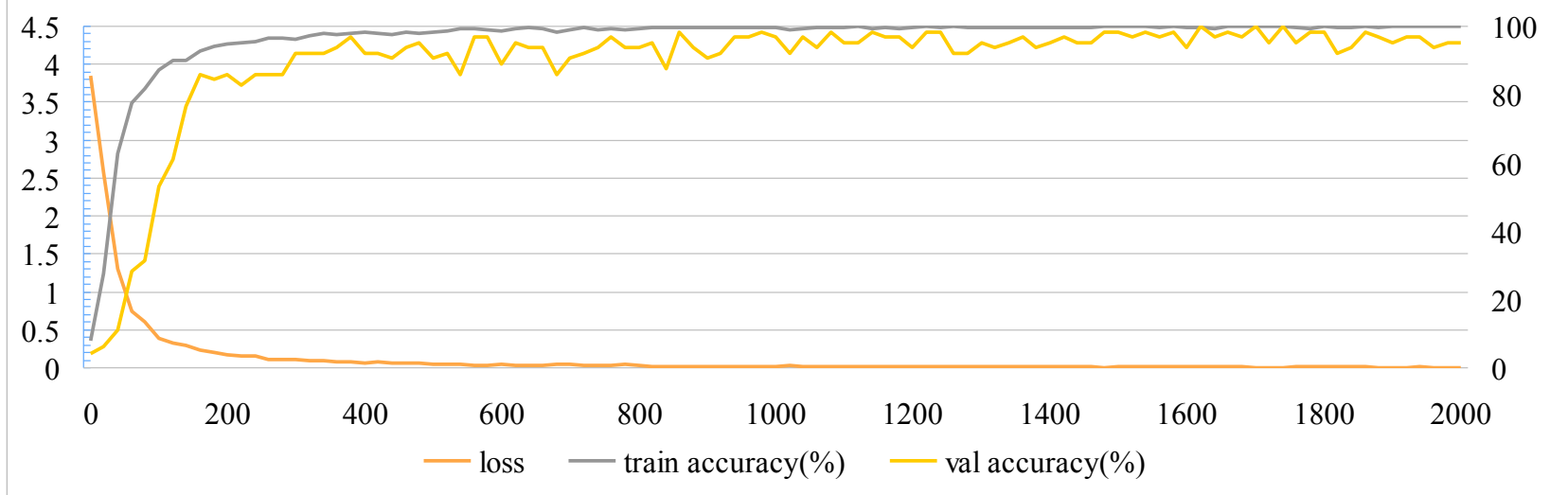

Fig. 8. Variation of train accuracy, test accuracy, and loss in training

The model accuracy on the training dataset reached $100 \%$, and the loss was 0.0005 after 4,000 iterations. Furthermore, the model accuracy on the verification dataset was $95.02 \%$, and the loss was 0.1434 .

\subsection{Testing and analysis}

Our rock image identification application was constructed on Android. The application was designed to acquire identified data by camera shooting and photo reading. The technical route is shown in Fig. 9. The CPU of the test platform was a 
Chunling Wang, Yan Li, Guangpeng Fan, Feixiang Chen and Wei Wang/

Journal of Engineering Science and Technology Review 11 (4) (2018) 111 - 117

Qualcomm Snapdragon 430. The output model size was 17.3 MB.

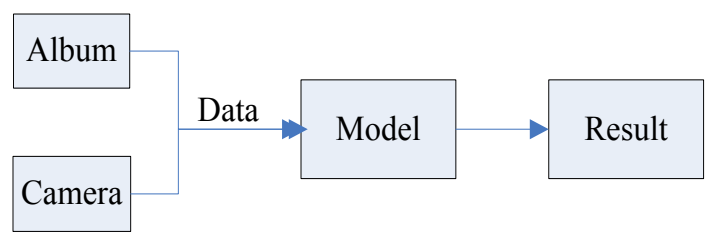

Fig. 9. Technical route

The test dataset and the trained model were input into the rock image identification application. Data were loaded and the model was operated; thus, test results were obtained. The confusion matrix is shown in Fig.10.

In the confusion matrix, the value of the middle row was the real value and the value of column was the predicted value. The accuracy of the entire test set reached $93.45 \%$. Over $91 \%$ of the test dataset were those images with $95 \%$ plus result confidence rate respectively. The single-result identification time distribution of the test dataset is shown in Fig. 11. Specifically, the single-result average identification time was $1,186 \mathrm{~ms}$. The maximum and minimum identification times were 1,409 and $858 \mathrm{~ms}$, respectively. The box plots showed no abnormal values, thereby indicating the stability of model identification.

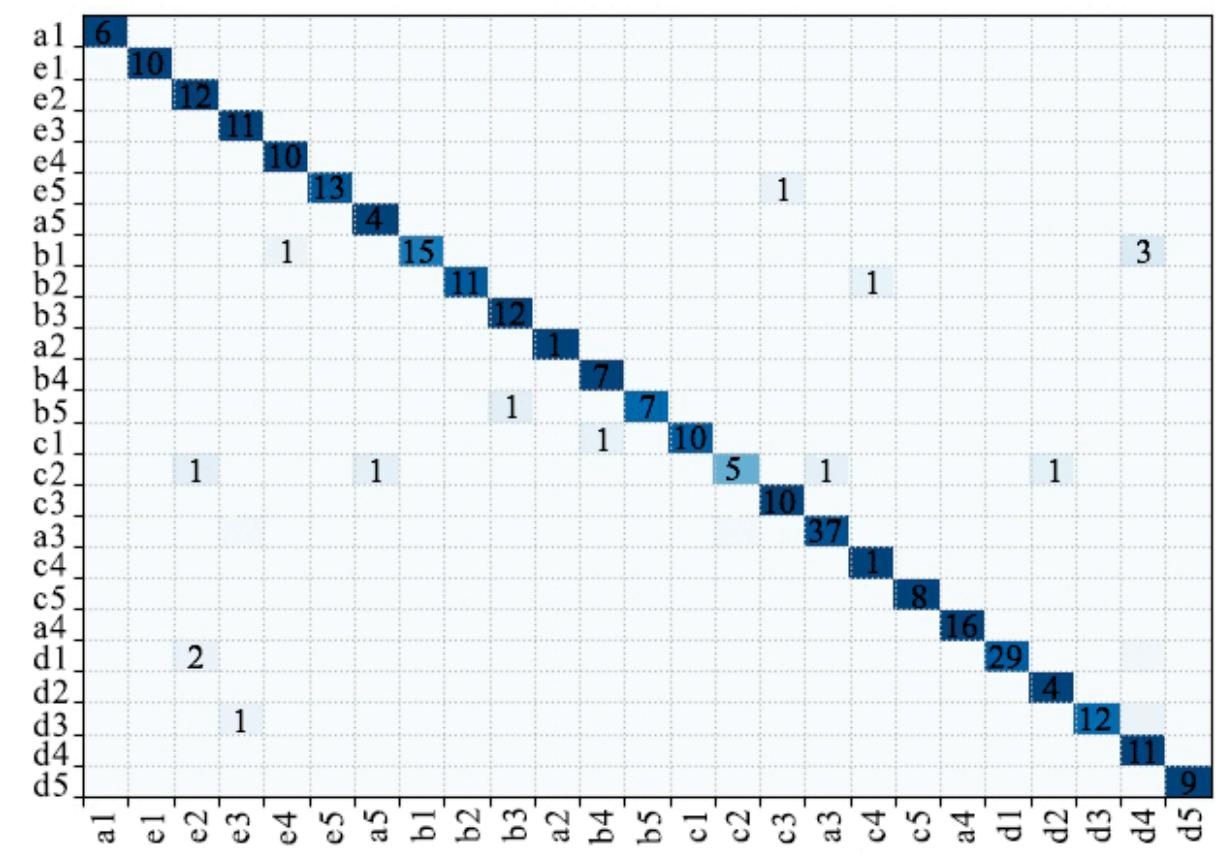

Fig. 10. Confusion matrix

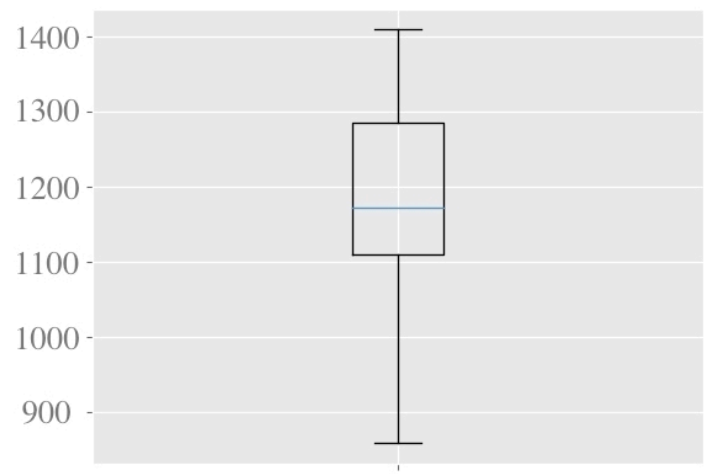

Fig. 11. Recognition time per image

\subsection{Testing and analysis}

To verify the applicability of the proposed method on the mobile terminal, the network ResNet50 with a standard convolutional structure was used to train, deploy, and test the rock image dataset. The comparison between ResNet50 and the proposed model are shown in Table 2.

Table 2. Results of contrast experiment

\begin{tabular}{c|c|c|c}
\hline Network & Test accuracy & Model size & $\begin{array}{c}\text { Single-result } \\
\text { recognition time }\end{array}$ \\
\hline ResNet50 & $92.76 \%$ & $94.7 \mathrm{MB}$ & $3050 \mathrm{~ms}$ \\
MobileNets & $93.45 \%$ & $17.3 \mathrm{MB}$ & $1186 \mathrm{~ms}$ \\
\hline
\end{tabular}

The contrast experiment demonstrated that given the similar accuracy of the test set, the model based on the depthwise separable convolution network had outstanding performances in model compression and computation. The model was applicable to fast accurate lithology identification of rock images under field offline conditions.

\section{Conclusions}

To achieve real-time fast feedback during field geological surveys, an automatic lithology identification model of rocks was proposed in this study on the basis of the depthwise separable convolutional structure and the transfer learning 
Chunling Wang, Yan Li, Guangpeng Fan, Feixiang Chen and Wei Wang/

Journal of Engineering Science and Technology Review 11 (4) (2018) 111 - 117

method. The pre-trained model of MobileNets was finetuned and then retrained on the selected dataset. The following conclusions were obtained.

(1) Compared with the standard convolutional network model, the depthwise separable convolutional network model shows fewer parameters, smaller size, higher computation speed, and shorter running time.

(2) The proposed method can accurately identify 25 types of rock images (e.g., light grey rhyolite). The model size is $17.3 \mathrm{MB}$ and the single-result average identification time is $1,186 \mathrm{~ms}$. The model is applicable to mobile devices. Experimental results demonstrate that the proposed method, which has short identification time and high reliability, is applicable to the fast accurate identification of rock images under field offline conditions.
Basing on the MobileNets with depthwise separable convolution and the transfer learning method, we proposed a new method for automatic identification of rock lithology in field geological survey. In future studies, the model identification capability can be improved by continuously enriching data of the training set. Quantization and compression are also available to further improve the performance of the model in mobile devices.

\section{Acknowledgements}

This work was supported by the Fundamental Research Funds for the Central Universities (Grant No.TD2014-02).

This is an Open Access article distributed under the terms of the Creative Commons Attribution License

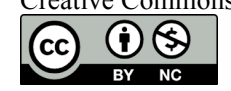

\section{References}

1. Li,C., Lv, X., Li, F., Li, J., Liu, C., "The architecture of geological survey intelligent space". Acta Geodaetica et Cartographica Sinica, 44(b12), 2015, pp.143-151.

2. Guo, C., Liu, Y., "Research on Rock Image Recognition in MultiColor Space". Science Technology and Engineering, 14(18), 2014, pp. 247-251.

3. Cheng, G., Liu L., "Feasibility study of deep learning algorithm applied to rock image processing”. Software Guide, 15(9), 2016, pp.163-166.

4. Cheng, G., Ma, W., Wei, X., Rong, C., Nan, J., "Petrofabric recognition based on image processing and neural network". Journal of Xi'an Shiyou University (Natural Science Edition), 28(5), 2013, pp.105-110.

5. Lepistö, L., Kunttu, I., Visa, A. , "Rock image classification using color features in Gabor space". Journal of Electronic Imaging, 14(4), 1005, pp.040503.

6. Singh, V., Rao, S. M., "Application of image processing and radial basis neural network techniques for ore sorting and ore classification". Minerals Engineering, 18(15), 2005, pp.1412-1420.

7. Singh, N., Singh, T, N, Tiwary, A., "Textural identification of basaltic rock mass using image processing and neural network". Computational Geosciences, 14(2), 2008, pp.301-310.

8. Cheng, G., Yang, J., Huang, Q., Liu, Y., "Classification and recognition of rock thin section images based on probabilistic neural network". Science Technology and Engineering, 13(31) 2013, pp.9321-9325.

9. Cheng, G., Yin, J., "Rock slice image classification based on SVM". Technological Innovation and Application, 2015(1), pp.38-38.

10. Zhang, C., Hao, L., Wang, Y., Zhang, Z.,"An image enhancement and lithology identification method based on Landsat8 OLI". Geology and Exploration, 53(2), 2017, pp. 325-333.

11. Li, X., "On-board pedestrian and cyclist recognition using deep learning methods". Master thesis of Tsinghua University, 2016.

12. Lin, M., " Research on face recognition based on deep learnin" Master thesis of Dalian University of Technology,2013.
13. Bianco, S., Buzzelli, M., Mazzini, D., Schettini, R., "Deep learning for logo recognition". Neurocomputing, 245(C), 2017, pp.23-30.

14. Schmidhuber, J., "Deep Learning in neural networks: An overview". Neural Networks, 61, 2015, pp.85-117.

15. Cheng, G., Guo, W., Fan, P., "Study on Rock Image Classification Based on Convolution Neural Network". Journal of Xi'an Shiyou University( Natural Science Edition), 32(4), 2017, pp.116-122.

16. Li, N., Hao, H., Gu, Q., Wang, D., Hu, X., "A transfer learning method for automatic identification of sandstone microscopic images". Computers \& Geosciences, 103, 2017, pp.111-121.

17. Zhang, Y., Li M., Han, S.,"Automatic identification and classification in lithology based on deep learning in rock images". Acta Petrologica Sinica, 34(2), 2017, pp.333-342.

18. Qureshi, A. S., Khan, A., Zameer, A., Usman, A., "Wind power prediction using deep neural network based meta regression and transfer learning". Applied Soft Computing, 58, 2017, pp.742-755.

19. He, K., Zhang, X., Ren, S., Sun, J., "Deep Residual Learning for Image Recognition". In: IEEE Conference on Computer Vision and Pattern Recognition, Las Vegas, USA: IEEE Computer Society, 2016, pp.770-778.

20. Sze, V., Chen, Y., Yang, T., Emer, J., "Efficient processing of deep neural networks: a tutorial and survey". Proceedings of the IEEE, 105(12), 2017, 2295-2329.

21. Pan, S J., Tsang I W., Kwok, J., Yang, Q., "Domain adaptation via transfer component analysis". IEEE Transactions on Neural Networks, 22(2), 2011, pp.199-210.

22. Pan, S. J., Yang, Q., "A Survey on Transfer Learning". IEEE Transactions on Knowledge \& Data Engineering, 22(10), 2010, pp.1345-1359.

23. Zeiler, M. D., Fergus, R., "Visualizing and Understanding Convolutional Networks". In: European Conference on Computer Vision. Springer, Cham, Germany: Springer, 2014, pp.818-833.

24. Wang, H., Chen, Y., Li, Y., "Face recognition method based on principal component analysis and Softmax regression model". Journal of Hefei University of Technology(Natural Science), 2015(6), pp.759-763. 